\title{
Mindfulness Promotes a More Balanced Time Perspective: Correlational and Intervention-Based Evidence
}

\author{
Michael Rönnlund $^{1}$ (D) Antonina Koudriavtseva ${ }^{1}$ - Linnea Germundsjö ${ }^{1} \cdot$ Terese Eriksson $^{1} \cdot$ Elisabeth Åström ${ }^{1}$. \\ Maria Grazia Carelli ${ }^{1}$
}

Published online: 22 February 2019

(C) The Author(s) 2019

\begin{abstract}
Objectives To investigate the relationship between mindfulness and aspects of time perspective (TP, i.e., habitual views of past, present, future).

Methods We examined cross-sectional associations between an established measure of mindfulness (FFMQ) and an extended version of the Zimbardo Time Perspective Inventory (Swedish ZTPI; S-ZTPI) in a sample with little experience of mindfulness training $(n=212)$. In addition, we evaluated the effects of two mindfulness-based interventions (a mindfulness instructor course involving 29 participants and a mindful self-compassion program, $n=40$ for the intervention group, $n=41$ for controls) on mindfulness and measures of TP including an aggregate measure of deviations from a proposed optimal, or balanced, time perspective (DBTP).

Results Cross-sectional data were consistent with a model by which part of the relationship between mindfulness and perceived stress is mediated by reduced DBTP. Global mindfulness scores showed the strongest (negative) associations with the S-ZTPI scales Future Negative and Past Negative. Comparisons of pre/post-intervention data revealed significant mindfulness-based intervention-related reductions of DBTP (Cohen's $d=-0.46$ ), with lowered scores on Past Negative and Future Negative and a small increase on Past Positive.

Conclusions The results support the notion that a higher level of mindfulness promotes a more balanced time perspective, with a reduced focus on negative aspects of the past and negative anticipations of the future. Relations to repetitive negative thought processes (rumination, worry) and a potential bidirectional influence of mindfulness and aspects of time perspective are discussed.
\end{abstract}

Keywords Mindfulness $\cdot$ Time perspective $\cdot$ Stress $\cdot$ MBI $\cdot$ Balanced time perspective

The way we perceive time, including the relative focus we put on the past, present, and future time frames, and our subjective evaluati on of these temporal frames, is referred to as time perspective (TP; Zimbardo and Boyd 1999). Individual differences in TP are assumed to develop rather early as the result of a habitual preference of one frame, or particular attitude, over the others (e.g., negative aspects of the past). Once crystallized, such temporal "biases" might be regarded as stable dispositions that increase vulnerability to mental ill health and

Michael Rönnlund

michael.ronnlund@umu.se

1 Department of Psychology, Umeå University, SE-90187 Umea, Sweden maladaptive behaviors (Zimbardo and Boyd 1999, 2008). A corollary to this position is that there is an optimal, or balanced, time perspective (BTP) that promotes subjective wellbeing and mental health (Zimbardo and Boyd 1999, 2008), an idea that sparked much recent interest (e.g., Mooney et al. 2017; Rönnlund et al. 2017; for a review, see Stolarski et al. 2015).

Efforts to operationalize BTP were mainly based on the Zimbardo Time Perspective Inventory (ZTPI; Zimbardo and Boyd 1999; for an alternative, see Webster and Ma 2013). ZTPI comprises five subscales. Past Positive captures a warm, positive, and nostalgic view of the past, whereas Past Negative reflects a negative and aversive view. The two scales that target the present time frame distinguish a live-for-themoment attitude involving immediate pleasure seeking without much concern of future consequences: Present Hedonistic, 
and a hopeless and helpless attitude toward the present: Present Fatalistic. Finally, Future captures a broad future orientation involving optimism, planning, and striving for future rewards. According to Zimbardo and Boyd (1999, 2008), BTP is characterized by high appreciation of the past, moderate to high levels of pleasure seeking, and a moderately pronounced future TP and lack of aversion toward the past or fatalistic attitudes toward the present. In addition, Zimbardo and Boyd suggested that BTP involves the ability to flexibly shift between time frames depending on situational demands.

To define BTP on the basis of the ZTPI score profile, some studies adopted a cluster-analytic approach (Boniwell et al. 2010; Seema and Sircova 2013) and others used a cutoffbased method (e.g., Drake et al. 2008), whereby certain ranges of scores on the ZTPI dimensions were regarded as criteria of BTP. The most commonly used method, though, is that referred to as deviations from BTP (DBTP; Stolarski et al. 2011). DBTP is computed as the aggregate difference between the observed and ideal ZTPI score profile (Stolarski et al. 2011; Zimbardo and Boyd 2008) which yields a continuous and approximately normally distributed score. This is convenient to the extent that the data are amenable to parametric statistical methods. DBTP also showed an advantage relative to alternative methods to operationalize BTP in regard to concurrent association with ratings of life satisfaction (Zhang et al. 2013), and more recent studies demonstrated substantial associations between DBTP and measures of happiness (Stolarski and Matthews 2016), perceived stress (Papastamatelou et al. 2015), severity of PTSD syndrome following motor vehicle accidents (Stolarski and CyniakCieciura 2016), and depressive symptoms (Mooney et al. 2017). Importantly, one limitation of the DBTP method is that it is based on the original ZTPI, which does not measure negative views or attitudes toward the future. A negative future TP might be particularly important to take into account in research of well-being or psychological distress. In an extended version of the ZTPI, the Swedish ZTPI (S-ZTPI; Carelli et al. 2011), this aspect is taken into account by a separate Future Negative scale. Based on this rationale, Rönnlund et al. (2017) suggested an alternative version of DBTP that also incorporates Future Negative. A direct comparison between DBTP with and without the inclusion of Future Negative indeed showed that inclusion of Future Negative within the DBTP operationalization was more strongly associated with well-being (Rönnlund et al. 2017) and with perceived stress (Rönnlund et al. 2018).

In recent years, BTP has been studied in relation to mindfulness. Mindfulness denotes a particular way of relating to time, by which attending to the present is considered essential to well-being (Kabat-Zinn 1990). Mindfulness, as a psychological process, involves bringing one's attention to experiences occurring in the present moment and more precisely defined as "non-elaborative, non-judgmental, present- centered awareness in which each thought, feeling, or sensation that arises in the attentional field is acknowledged and accepted as it is" (Bishop et al. 2004, p. 232), and as a trait, the ease to which a person attains this type of state. A wealth of studies confirm that higher levels of dispositional mindfulness, as reflected by measures such as the Five Facets of Mindfulness Questionnaire (FFMQ; Baer et al. 2006) and the Mindful Attention Awareness Scale (MAAS; Brown and Ryan 2003), are inversely related to a variety of distresses, including depressive symptoms, anxiety, stress, and positively associated with measures of happiness and life satisfaction (Keng et al. 2011).

Obviously, mindfulness constitutes quite a different way of relating to the present than is captured by either of the ZTPI dimensions but may rather be regarded as related to aspects of BTP (Stolarski et al. 2015). Indeed, Zimbardo and Boyd (2008) described a TP, which they called "holistic present," with explicit reference to Buddhist philosophy and mindfulness. Attending to the present in this particular sense, they argued, may support integration of aspects of the past and future and was described as a state of mind in which "...the past, the present, the future, the physical, the mental and the spiritual mental elements in life are not separate but closely interconnected within you." (Zimbardo and Boyd 2008, p. 110 ); a synchronistic process akin to the proposed dynamic aspect of BTP (Stolarski et al. 2015). Mello and Worrell (2015) used the term time relation to describe the degree to which one perceives that the past, present, and future are interrelated. Apart from these notions, a common reference to relations to time as essential to well-being, and common associations with major outcome variables (e.g., happiness and perceived stress), warrants attention to whether mindfulness and aspects of TP, including BTP, are associated.

Turning to empirical studies, the first (Drake et al. 2008) classified participants as either BTP or non-BTP using a cutoff at the 33rd or 66th percentile for the separate ZTPI scales to yield the constellation of scores regarded to reflect BTP (Zimbardo and Boyd 1999). Based on these criteria, 13 of the 260 participants were considered as having a BTP. Critically, the BTP group exhibited a higher mean score on MAAS compared with the non-BTP group. Analyses of separate ZTPI scales revealed a negative correlation between MAAS score and Past Negative $(r=-.49)$ and Present Fatalistic $(r=-.23)$. Additionally, a small but significant negative association with Present Hedonistic was observed, whereas MAAS score was unrelated to Future.

In a second study by Seema and Sircova (2013), scores on the five ZTPI scales, MAAS, and the measures of well-being were submitted to a cluster analysis that resulted in four ZTPI score profiles, one of which was deemed to match the criteria of BTP. A substantial difference in prevalence of the "BTP type" compared with the first study might be noted: $37.8 \%$ of the participants were classified as BTP, compared with $5 \%$ in 
Drake et al. (2008). This cluster was additionally characterized by higher MAAS scores and higher ratings of well-being, supporting the association between mindfulness and BTP. Analyses of subscales confirmed a negative association of mindfulness and Past Negative and Present Fatalistic. However, unlike the results of the study by Drake et al. (2008), Future was positively correlated with MAAS $(r=.23)$ and mindfulness was not associated with Present Hedonistic.

Wittmann et al. (2014) examined specific facets of mindfulness in relation to TP subscales based on the Freiburg Mindfulness Inventory (FMI; Walach et al. 2006) and the Comprehensive Inventory of Mindfulness Experiences (CHIME; Bergomi et al. 2013). The FMI and CHIME "acceptance" facets were negatively associated with Past Negative $(r s-.43$ to -.46$)$ and positively related to Past Positive. Thus, one mechanism, by which higher mindfulness might reduce TP biases and increase BTP, could be by improved ability to accept and disengage from negative aspects of one's past (and possibly also worry about the future). Past Negative was also associated CHIME "decentering" and "openness" ( $r \mathrm{~s}=-0.46$ to -0.38$)$ scales. Future was correlated with "Acting with Awareness" and "insight" $(r=.36$ and .42 ), which is interesting given the lack of association of Future and global mindfulness in Drake et al. (2008). Similarly, Wittmann et al. (2015a) reported a moderate positive association between FMI "presence" (the only mindfulness score included) and Present Hedonistic (but cf. Wittmann et al. 2014), a scale that was either unrelated to mindfulness or even negatively associated with mindfulness in other studies (e.g., Drake et al. 2008; Seema and Sircova 2013).

Finally, two recent studies (Stolarski et al. 2016; Muro et al. 2017) adopted DBTP instead of the coarse BTP/non-BTP categorization. In Stolarski et al. (2016), measures of mindfulness, DBTP score, and life satisfaction were submitted to mediation analyses. The results were consistent with a model by which mindfulness exerts an indirect influence on life satisfaction via reduced DBTP but were indicative of partial rather than complete mediation suggesting that minimized DBTP may be one of several means by which mindfulness promotes life satisfaction. Muro et al. (2017) similarly demonstrated a substantial correlation between mindfulness and DBTP in a sample of Catalan students and indicated that both DBTP and mindfulness (MAAS score) were related to life satisfaction.

Whereas prior studies showed that higher levels of dispositional mindfulness are related to BTP and a less aversive view of the past, it remains to be demonstrated whether mindfulness skills acquired by training may alter one's TP. Even though TP is considered a relatively stable individual differences variable, there is some evidence of the malleability of TP as such. For example, in time perspective therapy (Sword et al. 2014; Zimbardo et al. 2012), the focus is to reduce distress by reducing TP biases and work toward a $\mathrm{BTP}$, indicating that $\mathrm{TP}$ is, to some extent, a modifiable aspect of personality. A straightforward prediction based on the mediational analyses by Stolarski et al. (2016; cf. also Adshead 2013) would be that cultivating mindfulness skills should not only remedy symptoms of distress and promote life satisfaction (for meta-analyses, see Khoury et al. 2015; Spijkerman et al. 2016) but should also reduce temporal biases and DBTP. Reports of lower scores on Past Negative in experienced mindfulness meditators compared with non-meditators (Droit-Volet and Heros 2017; Wittmann et al. 2015b) seem to support such a pattern, but quasi-experimental evidence is not conclusive due to the potential influence of confounding variables. To further investigate the link between mindfulness and aspects of TP, the present study therefore examined cross-sectional associations of the constructs as well as effects of mindfulness-based interventions (MBIs) on measures of TP. We adopted the extended version of the Zimbardo scale (S-ZTPI) to differentiate between (positive/negative) aspects of future TP, which we expected to increase associations between mindfulness and DBTP. The possibility that different facets of mindfulness would be differently associated with separate aspects of TP was additionally attended to.

\section{Method}

\section{Participants}

Three samples were included. Sample 1 comprised 111 university students ( $M=25.9$ years, $S D=7.0 ; 70 \%$ females). Sample 2 involved 101 psychologists $(M=36.2$ years, $S D=$ $8.2 ; 96 \%$ women) who were part of a randomized controlled trial (RCT) study involving a 6-week online MBI. Sample 3 comprised 29 individuals $(M=43.7$ years, $S D=12.2 ; 28$ women) enrolled in a mindfulness instructor course. A majority of the participants reported post-secondary education and no/little prior experience with mindfulness training.

\section{Procedure}

Participants in sample 1 were recruited from undergraduate courses at Umeå University, Sweden, and responded to an online survey including the relevant instruments (FFMQ, SZTPI, Perceived Stress Scale (PSS); see "Measures"). Participants in sample 2 were recruited via a closed Facebook group for Swedish psychologists. Licensed psychologists or psychologists in post-graduate training working part or fulltime were invited. Those willing to participate were provided a unique code to the online questionnaires (FFMQ, S-ZTPI, PSS) and were randomly assigned to a training or control group. Participants in the training group were provided unique log-in information required to start the training, via e- 
mail. Seven weeks later, the participants (i.e., in the training group and in the control group) were requested to respond to the questionnaires a second time. Following completion of the second assessment, participants in the control group were provided free access to the online training program. Participants in sample 3 were recruited through courses aimed to train and certify mindfulness instructors held by Mindfulnesscenter AB. The participants received information about the study before the course started. During the first day of the course, those willing to participate were provided questionnaires to fill in (MAAS, S-ZTPI, PSS) and return in an envelope with prepaid postage. At the final day of the course, the participants were requested to complete the same questionnaires and return these as before.

Instructor Course/8-Week Mindfulness Program (MBI 1) The mindfulness instructor course involved 6 days of instructor led practice $(1+2+2+1$ days) over a period of 4 months and an online mindfulness training program ("Here and Now"; Schenström 2017a). The program is organized in eight steps involving two short 3-4 min lectures per step informing of the rationale of the exercise and a 20-30-min mindfulness exercise per day 6 days a week (audio files) for 8 weeks. In total, the online program corresponds to about $16 \mathrm{~h}$ of total training and involves standard mindfulness exercises such as the breathing anchor, body scan, sitting meditation, and a few yoga/movement exercises including several alternations of each exercise. The stepwise organization of the exercises means that a new exercise is available only once the prior exercise in the predetermined sequence of exercises has been completed. To be certified as an instructor, participants had to complete the online mindfulness training program (which could be verified electronically) and at the end of the course write a short thesis about their experiences of the mindfulness training. Certification also involved passing an oral exam and an evaluation of the participants' skills. All participants in the study passed the exams and were certified as instructors.

Mindful Compassion Program (MBI 2) A 6-week online program "Mindfulness and compassion with self and others" developed by Schenström (2017b) was used. The program encompasses $10 \mathrm{~h}$ of training: about $15 \mathrm{~min}$ of training per day, 6 days a week. The program is organized in six steps involving different types of exercises with guided instructions (auditory files). The stepwise organization of exercises means that a new exercise is available only once the prior exercise in the predetermined sequence of exercises has been completed. The program involves standard mindfulness/compassion exercises such as breathing anchor, body scan, loving kindness, and exercises of compassion with self and others. Following completion of each of the exercises, instruction of an everyday exercise to be performed during the day is given (different exercises for the six steps).

\section{Measures}

FFMQ A Swedish version of the FFMQ (Lilja et al. 2011; Baer et al. 2006) was used to assess dispositional mindfulness and specific facets of mindfulness. The items describe a particular state of mind (e.g., "I pay attention to sensations, such as the wind in my hair or sun on my face") and are rated on a 5-point Likert scale ranging from Never/almost never (coded as 1) to Most of the time (coded as 5). A sum score is usually taken to indicate the individuals' global level of mindfulness. The Swedish version of the questionnaire is slightly reduced and contains 29 of the 39 original items, reflecting five facets: (1) Non-reactivity to Inner Experience (six items), (2) Observing (noticing/attending to sensations /perceptions/thoughts/feelings; seven items), (3) Acting with Awareness (automatic pilot/concentration/non-distraction; five items), (4) Describing (labeling feelings/thoughts with words; six items), and (5) Non-judging of Experience (five items). High levels of internal consistency $(\alpha=0.75-0.85)$ were reported across the facets and for the total scale ( $\alpha=0.81$; Lilja et al. 2011; cf. Baer et al. 2008). Confirmatory factor analyses supported a hierarchical structure with four of the facets as first-order factors and a general mindfulness factor but indicated that the facet Observe was not a significant part of a global structure (Lilja et al. 2011). Validity evidence includes significant associations with meditation experience (Lilja et al. 2011), psychological symptoms, and measures of well-being (Baer et al. 2008).

MAAS A Swedish translation of the MAAS (Hansen et al. 2009; Brown and Ryan 2003) was used. MAAS is a 15-item measure of dispositional mindfulness, including receptive awareness of, and attention to, what takes place in the present. The items (e.g., "I find it difficult to stay focused on what's happening in the present"; "I rush through activities without being really attentive to them") are rated on a 6-point Likert scale ranging from Almost always (coded as 1) to Almost never (coded as 6). A higher total score (range 15-90) indicates a higher level of mindfulness. Acceptable levels of internal consistency was observed $(\alpha=0.77-0.86$ for the Swedish version; Hansen et al. 2009) with evidence that the scale reflects a unitary factor (Brown and Ryan 2003). Validity evidence included relations to self-rated health and selfesteem (Hansen et al. 2009) and prognostic validity of measures of self-regulation and well-being (Brown and Ryan 2003).

S-ZTPI (Samples 1-3) S-ZTPI (Carelli et al. 2011) consists of 64 items: the 56 items in the original ZTPI (Zimbardo and Boyd 1999) and 8 extra items assigned to a new Future Negative scale. The inventory comprises six temporal dimensions: Past Positive (PP; nine items, e.g., "Familiar childhood sights, sounds, smells often bring back a flood of wonderful 
memories"), Past Negative (PN; ten items, e.g., "Painful past experiences keep being replayed in my mind"), Present Hedonistic (PH; 15 items, e.g., "I believe that getting together with one's friends to party is one of life's important pleasure"), Present Fatalistic (PF; nine items, e.g., "Fate determines much in my life"); Future Positive (11 of the 13 items in the original Future scale, e.g., "When I want to achieve something, I set goals and consider specific means for reaching those goals"), and Future Negative (FN; ten items; two from the original Future and eight new, e.g., "The future contains too many boring decisions that I do not want to think about"). The participant is requested to rate each statement in regard to how characteristic it is of his/her own view on 5-point Likert scale ranging from Very uncharacteristic (coded as 1) to Very characteristic (coded as 5). Acceptable internal consistencies $(\alpha=0.70-0.84)$ and test-retest reliabilities ranging from 0.60 to 0.85 were observed across the subscales, including the new Future Negative scale (Carelli, et al. 2011). Validity evidence included associations with the General Decision Making Styles scale (Scott and Bruce 1995) with correlations of the S-ZTPI dimensions in expected directions, for example, a strong positive association between Future Negative and scores on a "dependent" style and a positive association between Future Positive and a "rational" style (Carelli et al. 2011).

DBTP based on S-ZTPI is referred to here as DBTP-e (to denote a difference from the formula for DBTP based on the original ZTPI, referred to DBTP-o) that was computed as follows (cf. Rönnlund et al. 2017; Stolarski et al. 2011):

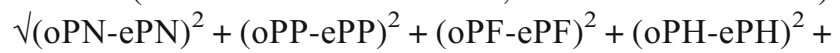
$(\mathrm{oFP}-\mathrm{eFP})^{2}+(\mathrm{oFN}-\mathrm{eFN})^{2}$, where o $=$ optimal score and $\mathrm{e}=$ empirical (i.e., observed) score. DBTP-o is computed following omission of FN and substituting scores for FP with scores for Future $(\mathrm{F})$, based on the 56 items in the original ZTPI. In line with previous studies (Rönnlund et al. 2017; Stolarski et al. 2011), optimal values for subscale scores were set to: $\mathrm{oPN}=1.95, \mathrm{oPP}=4.6, \mathrm{oPF}=1.5, \mathrm{oPH}=3.9, \mathrm{oF} / \mathrm{oFP}=4.0$, and $\mathrm{oFN}=1.8$. The ZTPI $o$ values were scores corresponding to specific percentile ranks in a cross-cultural database (see Stolarski et al. 2011). The value for FN was defined by the same percentile (10th) adopted for PN and PF in the former study (Rönnlund et al. 2017).

PSS A Swedish translation of the PSS (Eskin and Parr 1996; Cohen et al. 1983) was used. PSS includes 14 items describing experiences that life has been unpredictable, uncontrollable, and overly demanding during the last month. The participant rates each of the descriptions in regard to frequency of occurrence, from Never (coded as 0) to Very often (coded as 4). High levels of internal consistency ( $\alpha=$ 0.84-0.86) were reported across samples (Cohen et al. 1983; for a review of psychometric properties and criterion validity, see Lee 2012).

\section{Data Analyses}

Pearson's $r$ was used to examine bivariate associations. Hierarchic linear regression analyses were adopted to test models involving mindfulness and DBTP as predictors of stress, with stepwise entry of the predictors. Mediation analyses were conducted to test a hypothetical causal model involving mindfulness, DBTP, and stress. A bootstrap procedure involving 1000 bootstrap samples was employed to test direct and indirect effects with $95 \%$ confidence bias-corrected confidence intervals for estimates. To evaluate effects of the MBIs, independent $t$ tests and mixed 2 (group) $\times 2$ (time) analyses of variance (ANOVAs) were performed. IBM SPSS/AMOS 23 were used. To compute Cohen's $d$ in the pre-posttest-control group design (MBI 2), we used the formula recommended by Morris (2008; dppc2), computed as the mean pre-post change in the treatment group minus the mean pre-post change in the control group, divided by the pooled pretest standard deviation. Analyses of simple correlations (Pearson's $r$ ) and hierarchical regressions were finally used to examine association of scores at baseline and association of change scores across the measures.

\section{Results}

We first tested a hypothetical mediational model by which the relationship between mindfulness and perceived stress is driven by reduced DBTP. Prior to testing this model, stepwise hierarchic regression analyses involving FFMQ total score and DBTP as the predictors of PSS score were performed (cf. Stolarski et al. 2016). The variations in DBTP measure (DBTP-o, vs. DBTP-e) were considered in separate regression analyses. The results for sample 1 and sample 2 yielded highly similar estimates and a virtually identical difference between DBTP-e and DBTP-o. Hence, data for the combined samples $(n=212)$ are reported here and in subsequent analyses.

FFMQ score entered as a predictor in step 1 accounted for $31.9 \%$ of the variance in PSS score. In step 2, DBTP score added significant variance, with a larger value increment in $R^{2}$ for DBTP-e $\left(\Delta R^{2}=.12\right)$ compared with DBTP-o $\left(\Delta R^{2}=.07\right)$. This yielded total $R^{2}$ values of 0.44 and 0.38 , respectively, for the model involving DBTP-e and DBTP-o. Reversing the order of entry of the predictors showed that DBTP entered in step 1 was a significant predictor of PSS score $\left(R^{2}=.18\right.$ for DBTP-o; $R^{2}=.31$ for DBTP-e) and that FFMQ entered in step 2 accounted for additional variance $\left(\Delta R^{2}=.20\right.$ entered after DBTP-o; $\Delta R^{2}=.13$ entered after DBTP-e). Thus, the analyses confirmed that FFMQ and DBTP scores were each significant predictors of perceived stress and also that they shared variance in predicting PSS score, each of the measures predicting unique amounts of variance in perceived stress. 
Next, the mediational model was configured in form of a path-analytic model in which mindfulness was considered as a predictor of perceived stress (direct effect) and of DBTP, with a potential indirect effect on stress via reduced DBTP. Once more, DBTP-o and DBTP-e were analyzed separately to estimate the potential value of differentiating positive/negative aspects of the future TP.

Figure 1 summarizes the model and the results of the analyses in form of standardized estimates ( $\beta$-weights) for the hypothetical paths: $\mathrm{a}=$ Mindfulness to DBTP, $\mathrm{b}=$ DBTP to stress, and $\mathrm{c}=$ direct effect of mindfulness on stress (i.e., not shared with DBTP), where c' represents the total (i.e., unmediated direct) effect of mindfulness on stress. Values for $\beta$ a13 pertain to a model including DBTP-o, whereas b1-3 pertain to a model including DBTP-e.

Regardless of the variation in DBTP measure, all three paths $(\mathrm{a}-\mathrm{c})$ were significant. Hence, the results were consistent with the hypothesis that (a) higher levels of trait mindfulness result in lower DBTP score, (b) higher DBTP score is predictive of higher levels of perceived stress, and (c) the relationship between mindfulness and stress is (in part) mediated by DBTP. Support of partial and not complete mediation is evident from a lower value for c compared with c' but a remaining significant effect of mindfulness on stress (i.e., the c-path is significantly different from zero even when DBTP is considered in the model). A further evaluation of the direct $(a=>$ $\mathrm{b}, \mathrm{b}=>\mathrm{c}, \mathrm{a}=>\mathrm{c})$ and indirect $(\mathrm{a}=>\mathrm{b}=>\mathrm{c})$ effects using the bootstrap method confirmed that the effects were significant (i.e., the lower limit of the $95 \%$ bias-corrected confidence intervals was larger than zero).

Additionally, the $\beta$ values were clearly higher for DBTP-e compared with DBTP-o. This held true for the path from mindfulness to DBTP as well as well as for the path from DBTP to stress. These patterns, in turn, resulted in a lower estimate for the c-path for DBTP-e compared with DBTP-o. In other words, differentiation of the future yielded a measure of DBTP that was more strongly associated with the global mindfulness score as well as the scores on the measure of perceived stress. Consequently, the new measure (DBTP-e) mediated more of the relationship between mindfulness and perceived stress than the original DBTP measure.

To examine the extent to which the global FFMQ score was differentially associated with separate S-ZTPI dimensions, and to provide information of which particular FFMQ facets were associated with each TP dimension, zero-order correlations were computed. These are summarized in Table 1.

Beginning with total FFMQ score, significant negative associations with Past Negative $(r=-.43, p<.001)$, Present Fatalistic $(r=-.24, p<.001)$, and Future Negative $(r=$ $-.53, p<.001)$ were observed. The values for the two versions of the DBTP measure differed significantly with higher values for DBTP-e compared with DBTP-o. As concerns the Non-reactivity facet, associations were significant for the three negatively valenced S-ZTPI subscales, and particularly strong for Future Negative $(r=-.53)$. Notably, the facet Observing showed no significant association with the S-ZTPI dimensions, except Future Positive $(r=.16, p<.05)$. In turn, Acting with Awareness was related to the three negatively valenced scales, with a small association for Present Fatalistic and moderate for Past Negative and Future Negative. The facet Describe showed a similar pattern but in addition a significant correlation with Future Positive $(r=.30)$. The facet Non-judging correlated negatively with the three negatively valenced S-ZTPI and additionally exhibited a small positive association $(r=.18, p<.01)$ with Past Positive. Neither FFMQ total nor any of the facets were associated with Present Hedonistic ( $r \mathrm{~s}=-.13$ to $.07, p \mathrm{~s}>.05$ ).

Apart from relationships of facets of mindfulness and separate TP dimensions, we examined correlations between ZTPI dimensions perceived stress (PSS score). The results showed that PSS score was strongly associated with Past Negative $(r=.53, p<.001)$ with a higher value still $(r=.67, p<.001)$

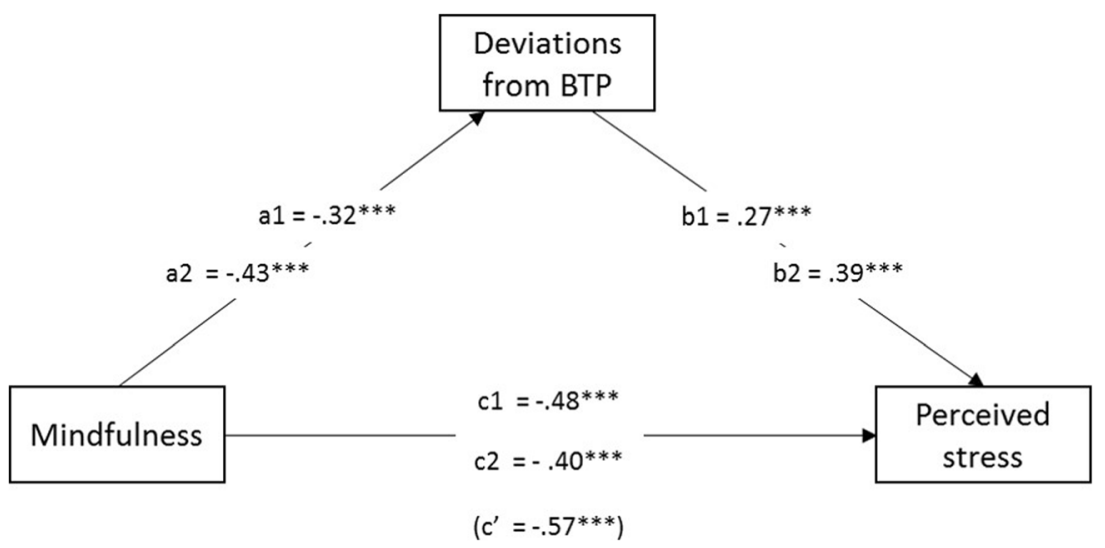

Fig. 1 Summary of mediational analyses of mindfulness (FFMQ score), deviations from BTP (DBTP score), and perceived stress (PSS-14 score). For each path, the values are presented as standardized coefficients. Values a1 to $\mathrm{c} 1$ are for the measure of DBTP based on the five original

ZTP dimensions (DBTP-o), whereas values a2-c2 are for the measure of DBTP (DBTP-e) computed on basis of the extended six-dimensional SZTPI. c' is the estimate of the total effect of mindfulness on perceived stress 
Table 1 Correlations of FFMQ total/five facets, ZTPI dimensions, and two measures of DBTP (DBTP-o based on the original five dimensional ZTPI and DBTP-e based on the six dimensional S-ZTPI)

\begin{tabular}{|c|c|c|c|c|c|c|c|c|c|}
\hline \multirow[t]{2}{*}{ Variable } & \multicolumn{6}{|c|}{ S-ZTPI dimension } & \multicolumn{3}{|c|}{ DBTP measure } \\
\hline & $\begin{array}{l}\text { Past } \\
\text { Positive }\end{array}$ & $\begin{array}{l}\text { Past } \\
\text { Negative }\end{array}$ & $\begin{array}{l}\text { Present } \\
\text { Hedonistic }\end{array}$ & $\begin{array}{l}\text { Present } \\
\text { Fatalistic }\end{array}$ & $\begin{array}{l}\text { Future } \\
\text { Positive }\end{array}$ & $\begin{array}{l}\text { Future } \\
\text { Negative }\end{array}$ & DBTP-o & DBTP-e & $\operatorname{Diff}(Z)$ \\
\hline FFMQ total & $0.15^{*}$ & $-0.44 * * *$ & 0.01 & $-0.24 * * *$ & 0.12 & $-0.53 * * *$ & $-0.32 * * *$ & $-0.43 * * *$ & $5.4 * * *$ \\
\hline F1 Non-react & 0.12 & $-0.35 * * *$ & 0.07 & $-0.15^{*}$ & -0.01 & $-0.58 * * *$ & $-0.25 * * *$ & $-0.36^{* * *}$ & $5.3 * * *$ \\
\hline F2 Observe & 0.01 & -0.06 & 0.06 & -0.06 & $0.16^{*}$ & -0.09 & -0.08 & -0.11 & 1.4 \\
\hline F3 Act Aware & 0.08 & $-0.33 * * *$ & -0.13 & $-0.18^{* *}$ & 0.04 & $-0.42 * * *$ & $-0.17^{*}$ & $-0.27 * * *$ & $4.7 * * *$ \\
\hline F4 Describe & 0.12 & $-0.31 * * *$ & -0.04 & $-0.26^{* * *}$ & $0.30 * * *$ & $-0.33 * * *$ & $-0.29 * * *$ & $-0.34 * * *$ & $2.4 * * *$ \\
\hline F5 Non-judge & $0.18^{* *}$ & $-0.48 * * *$ & 0.02 & $-0.18 * *$ & -0.09 & $-0.54 * * *$ & $-0.32 * * *$ & $-0.43^{* * *}$ & $5.5 * * *$ \\
\hline
\end{tabular}

$\operatorname{Diff}(Z) z$ test of the difference in $r$ value

$* p<.05 ; * * p<.01 ; * * * p<.001$

for Future Negative. A moderate association with Present Fatalistic $(r=.33, p<.001)$ was observed. By contrast, neither Present Hedonistic nor Future Positive showed a tendency of relationship with stress ( $r$ s .05, .04). Finally, DBTP-e showed a higher correlation with stress $(r=.56, p<.001)$ than DBTPo $(r=.43, p<.001, z=6.9, p<.001$ for the difference in $r$ value).

\section{MBI 1-Mindfulness Instructor Course}

Turning to the evaluation of effect of MBIs and data pertaining to the instructors in training (MBI 1), a significant increase in MAAS score was observed from the pre $(M=$ 55.41, $S D=10.68)$ to post-intervention $(M=67.77, S D=$ $7.15, t(28)=-6.30, p<.001)$. Scores on PSS were reduced $(M=24.11, S D=8.64$ prior to the course vs. $M=15.00, S D=$ 5.42 after the course), $t(28)=-.6 .30, p<.001$. The effect sizes were large (Cohen's $d>0.80$, Cohen 1988; $d=1.33$ for MAAS and $d=-1.14$ for PSS score). Of interest to note is also a significant correlation of pre-assessment MAAS and DBTP scores similar to that reported before $(r=-.49$, $p<.01)$.

Next, we attended to the critical comparison of ZTPI measures before and after the course. The data are summarized in Table 2, including means $(S D)$ observed before and after the intervention, result of dependent $t$ tests, and values for Cohen's $d$. The results revealed a reduction of DBTP-e score from the first $(M=2.43)$ to the second assessment $(M=2.11) p<.01, d=0.49$. A pattern of reduced scores was additionally observed for Past Negative and Future Negative ( $p s<.001, d=-0.43$ and -0.48 , respectively). A smaller but statistically significant decrease $(p=.02$; $d=0.32$ ) was observed for Present Fatalistic, while scores on Past Positive exhibited a mean increase from the pre to post assessment ( $p<.05, d=0.27)$. No tendency of pre- to post-difference was, by contrast, observed for Present Hedonistic or Future Positive ( $p$ values $>.70)$.

\section{MBI 2-Mindful Compassion Training}

The first MBI lacked a control group. Hence, potential influences of history, spontaneous remission, and instrument reactivity are threats to the internal validity. The second MBI controlled for such effects by virtue of the RCT design. Also, the somewhat larger sample size $(n=40$ in the training group, $n=$ 41 in the control group with complete pre/post assessment data) increased chances of evaluating small effects. Attrition analyses (20 dropouts vs. 81 returnees) revealed no significant differences between returnees and dropouts on any of the study variables. Beginning with FFMQ total score, a mean increase from the pre- $(M=3.13, S D=0.45)$ to postassessment $(M=3.51, S D=0.44 ; d=0.60)$ was observed in the training group with little change for the control group. PSS scores showed a selective decrease in the training group $(M=$ 25.28, $S D=6.99$ before vs. $M=19.43, S D=6.42$ after training, $d=-0.59)$.

The pre-post-TP data for the training group and the control group are present in Table 3. Effect size estimates (Cohen's $d$; Morris 2008) for the training group and the control group are also provided as well $F$ values pertaining to the time-by-group interaction term obtained in the ANOVAs across the TP measures. The results revealed a significant reduction in DBTP score $(p<.01, d=0.54)$. Moreover, scores on Past Negative $(d=0.39)$ and Future Negative $(d=0.52)$ were significantly reduced following training as in the previous MBI. In addition, Past Positive increased from the pre- to post-intervention assessment, with no difference in the control group. Present Fatalistic decreased in the training group before, but not after a Bonferroni adjustment. Also, inspection of the means for pre and post assessment showed a similar trend for the control group, which presumably account for the lack of a significant time-by-group interaction for this S-ZTPI dimension. Otherwise, as indicated by significant $F$ values (rightmost column), results of the ANOVAs further substantiated the aforementioned patterns of selective changes in DBTP, Past 
Table 2 Summary of S-ZTPI measures (M, SD) before and after the mindfulness instructor course $(n=29)$

\begin{tabular}{|c|c|c|c|c|c|c|c|}
\hline \multirow[t]{2}{*}{ Measure } & \multicolumn{2}{|c|}{ Pre assessment } & \multicolumn{2}{|c|}{ Post assessment } & \multirow[t]{2}{*}{$t$ value } & \multirow[t]{2}{*}{$p$} & \multirow[t]{2}{*}{$d$} \\
\hline & $M$ & $S D$ & $M$ & $S D$ & & & \\
\hline DBTP-e & 2.43 & 0.74 & 2.11 & 0.47 & $-2.77 * *$ & .009 & -0.49 \\
\hline Past Positive & 3.36 & 0.57 & 3.51 & 0.56 & $2.14 *$ & .047 & 0.27 \\
\hline Past Negative & 2.55 & 0.73 & 2.20 & 0.56 & $-4.02 * * *$ & $<.001$ & -0.51 \\
\hline Present Hedonistic & 3.06 & 0.52 & 3.06 & 0.52 & 0.05 & .961 & 0.03 \\
\hline Present Fatalistic & 2.39 & 0.53 & 2.23 & 0.46 & $2.56^{*}$ & .016 & 0.32 \\
\hline Future Positive & 3.49 & 0.49 & 3.43 & 0.45 & -1.18 & .300 & -0.12 \\
\hline Future Negative & 2.67 & 0.61 & 2.34 & 0.56 & $3.75 * * *$ & $<.001$ & -0.53 \\
\hline
\end{tabular}

$* p<.05 ; * * p<.01 ; * * * p<.001$, two-tailed test
Positive, Past Negative, and Future Negative in the training group.

Finally, we considered the combined data for MBI 1 and 2 (i.e., the training group; total $n=69$ ). Difference pre- vs. postintervention were significant at $p<.001$ for DBTP $(d=-$ 0.46). At the level of individual subscales, Past Negative $(d=-0.44)$, Past Positive $(d=0.28)$, and Future Negative $(d=-0.48)$ showed significant changes $(p<.001)$. Present Fatalistic was also reduced $(p=.002 ; d=-0.27)$. The foregoing effects remained following a Bonferroni adjustment controlling for multiple comparisons (i.e., $\alpha$ level 0.05/5 =0.01). No pre- vs. post-intervention difference was observed for Present Hedonistic $(p=.87 ; d=-0.01)$ and Future Positive $(p=.43 ; d=-0.05)$.

\section{Discussion}

The objective of this study was to examine relations between measures of mindfulness and TP, including aggregate measures of temporal balance (DBTP) and scores on the separate S-ZTPI dimensions. In addition to correlational analyses, effects of two MBIs were evaluated to test the hypothesis that improved mindfulness skills that reduce DBTP. The results of the cross-sectional analyses will be addressed first, followed by a discussion of the results from the MBIs. Finally, we consider relations to mechanisms through which mindfulness may influence mental health (Coffey et al. 2010).

\section{Dispositional Mindfulness and Time Perspective}

The results of correlational analyses were consistent with prior studies in several respects. First, dispositional mindfulness was inversely related to DBTP (Muro et al. 2017; Stolarski et al. 2016). Second, the mediational analyses were consistent with the hypothesis that part of the relationship between mindfulness and perceived stress is mediated by DBTP, much in line with the previous study of well-being by Stolarski et al. (2016). As was pointed out by Stolarski and co-workers, and reiterated here, the finding of partial rather than complete
Table 3 Summary of S-ZTPI measures at the pre- and postassessments in the training group and in the control group

\begin{tabular}{|c|c|c|c|c|c|c|c|c|c|c|}
\hline \multirow[b]{3}{*}{ Measure } & \multicolumn{4}{|c|}{ Training group $(n=40)$} & \multicolumn{4}{|c|}{ Control group $(n=41)$} & \multirow[b]{3}{*}{$d$} & \multirow[b]{3}{*}{$\begin{array}{l}G \times T \\
(F \text { value })\end{array}$} \\
\hline & \multicolumn{2}{|l|}{ Pre } & \multicolumn{2}{|l|}{ Post } & \multicolumn{2}{|l|}{ Pre } & \multicolumn{2}{|l|}{ Post } & & \\
\hline & $M$ & $S D$ & $M$ & $S D$ & $M$ & $S D$ & $M$ & $S D$ & & \\
\hline DBTP-e & 2.46 & 0.70 & $2.16^{* * *}$ & 0.78 & 2.49 & 0.73 & 2.58 & 0.80 & 0.54 & $18.60^{* * *}$ \\
\hline Past Positive & 3.51 & 0.59 & $3.68 * *$ & 0.60 & 3.54 & 0.64 & 3.49 & 0.74 & 0.36 & $8.82 * *$ \\
\hline Past Negative & 2.55 & 0.58 & $2.33 * * *$ & 0.64 & 2.82 & 0.75 & 2.86 & 0.78 & 0.39 & $10.77 * *$ \\
\hline Present Hedonistic & 2.91 & 0.46 & 2.89 & 0.53 & 3.12 & 0.52 & 3.05 & 0.55 & 0.10 & 1.04 \\
\hline Present Fatalistic & 2.48 & 0.51 & $2.34^{*^{\mathrm{a}}}$ & 0.37 & 2.43 & 0.49 & 2.37 & 0.52 & 0.16 & 0.17 \\
\hline Future Positive & 3.55 & 0.45 & 3.54 & 0.45 & 3.52 & 0.46 & 3.51 & 0.53 & 0.00 & 0.05 \\
\hline Future Negative & 2.91 & 0.53 & $2.65 * * *$ & 0.58 & 2.85 & 0.62 & 2.89 & 0.57 & 0.52 & $10.20 * *$ \\
\hline
\end{tabular}

$D B T P$ Deviations from a Balanced Time Perspective (extended formula), $G \times T$ group-by-time interaction ${ }^{*} p<.05 ; * *<.01$; *** $p<.001$; asterisks for post assessment scores pertain to level of significance of the pre-/ post-difference as determined by dependent $t$ tests (two-tailed)

${ }^{\mathrm{a}}$ No longer significant following Bonferroni adjustment (alpha level $=0.025$ ) 
mediations indicates that TP biases reflected by the DBTP is not the only path through which mindfulness exerts an influence on the outcome variable, i.e., perceived stress. Similarly, in the regression analyses of perceived stress, mindfulness and DBTP accounted for unique variance. Nonetheless, the analyses provided additional support of the notion that mindfulness and BTP/DBTP share a substantial amount of variance and were consistent with the idea that higher levels of dispositional mindfulness are accompanied by a more balanced TP, which in turn may reduce stress.

As concerns the separate S-ZTPI dimensions, global mindfulness (FFMQ) score was negatively associated with Past Negative and Present Fatalistic but unrelated to Present Hedonistic, in line with prior studies (Drake et al. 2008; Muro et al. 2017). A significant positive correlation between global mindfulness score and Past Positive was additionally observed (Drake et al. 2008; Muro et al. 2017; but see Seema and Sircova 2013). No significant association between FFMQ total score and Future Positive was observed, consistent with Drake et al. (2008, i.e., for Future), whereas, presumably due to the large sample, the corresponding correlation, of the same magnitude as the one observed in the present study $(r=.12$; for MAAS and Future scores), was significant in Muro et al. (2017). Hence, as concerns the five original ZTPI dimensions, the results were largely consistent with prior research, showing that higher dispositional mindfulness concurs with less aversion toward the past in particular and lower degree of fatalistic attitudes toward the present.

A novel aspect of the results concerned the relationship between mindfulness and Future Negative, a TP dimension not considered in prior studies. In fact, Future Negative turned out to be the single S-ZTPI dimension that exhibited the strongest (negative) association with the measure of global mindfulness. As a result, the extended DBTP measure taking the Future Positive/Negative distinction into account was, as we had expected, more closely linked to mindfulness than the original DBTP measure (Stolarski et al. 2011). The observation that Future Negative was strongly correlated with perceived stress is consistent with the results of another recent study (Rönnlund et al. 2018). Hence, the present results provide further support that a future negative TP dimension is important to consider to account for variations in behaviors and mental health factors (e.g., Blomgren et al. 2016; Carelli et al. 2011; Rönnlund et al. 2017; Stolarski and Matthews 2016).

Analyses of the separate FFMQ facets were moreover consistent with results in Wittmann et al. (2014), to the extent that Non-judging of Experience, in common with the similar acceptance-dimensions of the FMI and CHIME scales in Wittmann et al., showed a substantial inverse association with Past Negative. Non-judging of Experience was moreover strongly negatively correlated with Future Negative and associated with lower scores also on Present Fatalistic. Much the same pattern was observed for Non-reactivity to Inner Experience, i.e., inverse associations with Past Negative, Present Fatalistic, and scores on Future Negative in particular. The strong association between Future Negative and the Nonreactivity seems consistent with findings of a link of Future Negative to anxiety ( $\AA$ ström et al. 2014) and a relationship between Non-reactivity and anxious arousal observed in other studies (e.g., Desrosiers et al. 2013). Acting with Awareness, a core component in definitions of mindfulness, was, in turn, negatively associated with all three negatively valenced SZTPI dimensions, especially Future Negative, suggesting that staying focused on present-moment experience and acting deliberately concurs with less aversion toward the past, lower present fatalism, and negative expectations of the future. The facet Observing was, by contrast, not associated with any of the S-ZTPI dimensions, except a small association with Future Positive, possibly reflecting the fact that this facet is less reflective of a general mindfulness factor in non-mediators (Aguado et al. 2015). Future Positive additionally exhibited a moderate positive association with the FFMQ facet Describe, involving the ease at which one describes feelings or thoughts with words, despite the lack of association with total FFMQ score. Presumably, these skills concur with aspects of Future Positive, for example, planning and goal setting that relate to the ability to verbalize thoughts and feelings. Once more, the three negatively valenced TP dimensions showed an inverse relationship with the foregoing facet, meaning that a general negativity is associated with difficulties in this regard.

Finally, none of the mindfulness facets were correlated with Present Hedonistic, in line with the relative lack of association with global mindfulness observed here and in prior studies. Interestingly, the inclusion of a eudemonic present scale reflecting mindfulness and flow increased the predictive validity of an alternative measure of BTP (based on the relative focus on the past and future; Vowinckel et al. 2017). Possibly, ZTPI would benefit from broadening the measurement of the present time perspective, so as to include a scale targeting eudemonic aspects. Indeed, a recent effort was made to establish such a new scale called Expanded Present scale (Zimbardo et al. 2017).

\section{Effects of Mindfulness-Based Interventions}

Turning to the effects of MBIs, we demonstrated that such interventions promote a more balanced TP. Significant reductions in DBTP were evident following both MBIs and were comparable in regard to effect size. As concerns the separate S-ZTPI dimensions, the results were in general consistent with a hypothesis that the dimensions exhibiting the strongest association with dispositional mindfulness would also be more likely to be show MBI-related changes. More specifically, scores on Past Negative were significantly reduced after the 
MBIs, in line with the negative association of this scale with dispositional mindfulness (see also Muro et al. 2017; Seema and Sircova 2013; Stolarski et al. 2016; Wittmann et al. 2014). The result is also consistent with findings of lower Past Negative in meditators compared with non-meditators (Droit-Volet and Heros 2017; Wittmann et al. 2015b). In a similar vein, reduced scores on Past Positive were observed following the MBIs (cf. Drake et al. 2008; Muro et al. 2017). Thus, mindfulness training appears to have a dual effect to ameliorate one's view of the past: reducing the negative aspects and boosting the positive. Presumably, increased mindfulness skills (e.g., non-judgment of experience) serve to reduce tendencies to dwell on aversive experiences in the personal past. This might in turn support retrieval of more pleasant events by making them more accessible and salient in memory. Given evidence that MBIs reduce negative affect (Khoury et al. 2015) and promotes positive affect (Garland et al. 2015), the dual effect of MBIs on past TP also seems consistent with the principle of mood-congruent retrieval of autobiographical memories (Teasdale and Fogarty 1979).

Following both MBIs, reduced scores on Present Fatalistic were moreover observed, in line with the cross-sectional pattern of a negative association between this scale and global mindfulness. The fact that the control group (in the second MBI) showed a similar pattern complicates interpretation of the effect, though. Hence, future studies need to determine whether mindfulness training, as we would expect, may also serve to reduce present fatalistic attitudes. No effect of mindfulness training on Future Positive was observed, much in line with the lack of association between Future/Future Positive here and in prior studies. By contrast, scores on Future Negative were markedly reduced by the training. This further underscores a need to consider negative as well as positive aspects of a future time perspective.

In terms of effect size, the changes in TP following the MBIs were in the upper range of a small effect for DBTP $(d=-0.46$, overall), Past Negative $(d=-0.43)$, and Future Negative $(d=-0.48)$, with a smaller effect for Past Positive $(d=0.28)$. These changes are nonetheless noteworthy given notions that TP biases are trait-like and stable. The present evidence of modifiability of TP biases is not necessarily incompatible with that of trait like properties, though. Follow-up analyses of data in the larger intervention group (MBI 2) indeed demonstrated a very high correlation of pre- and postintervention DBTP scores $\left(r_{\mathrm{tt}}=.81\right.$, higher still in the control group: $r_{\mathrm{tt}}=.89$ ). Thus, high rank-order stability of DBTP was observed despite a general pattern of reduced DBTP scores, indicating that modifiability was paired with considerable preservation of pre-existing (trait-like) inter-individual differences in DBTP. The modifiable aspect certainly deserves to be highlighted here, especially given that the changes were observed following rather brief interventions. As such, the present observation of significant modifiability of a construct regarded to have a substantial stable (trait-like) component is in parallel with other findings in the psychological literature. Indeed, the modifiability of scores on mindfulness measures (considered to reflect trait mindfulness) is an example of such a pattern.

\section{Influence of Mindfulness on Mental Health via Changes in Time Perspective}

Correlational data and results from two MBIs provided converging evidence of a substantive link between mindfulness and TP. The particularly strong inverse association of the FFMQ facets "Non-judging of Experience" and "Non-reactivity to Inner Experience" to Past Negative, and Future Negative, especially, is interesting to consider in relation to paths through which mindfulness may support well-being and reduce psychological distress (Coffey et al. 2010) in part by modifying aspects of one's TP.

The first path might be through enhancing emotion regulation, more specifically, by improving one's ability to adaptively cope with negative emotions. In this regard, the aforementioned patterns of results suggest that being mindful, particularly in terms of an accepting or non-judgmental attitude and less reactivity to inner experiences, might reduce psychological distress by decreasing negative emotional reactivity emanating both from a focus on negative aspects of the past and/or negative anticipation of the future.

The present result also seem relevant to a second proposed path, namely modulation of persistent negative thought processes such as rumination and worry (Coffey et al. 2010, referred to both as rumination). More specifically, rumination and worry may be regarded as differentially temporally oriented thought process (e.g., Watkins et al. 2005) such that rumination is past-oriented (cf. Past Negative) and worry future-oriented (cf. Future Negative). Furthermore, rumination (Coffey et al. 2010) and worry (Janowski and Lucjan 2012) exhibited similar inverse associations with an "acceptance" factor (cf. Non-judging) as Past Negative and Future Negative. Hence, lowered rumination and worry could possibly be a mediator in the link between mindful acceptance, Past Negative and Future Negative, and psychological distress, a hypothesis that needs to be examined in future studies.

Notwithstanding the present demonstration of reduced TP biases following mindfulness-based interventions, a reversed influence, i.e., from TP to mindfulness, is worth considering. In particular, Past Negative or Future Negative, and related negative thought processes (rumination/worry), may act as obstacles to a more mindful attitude. Rumination and worry at least appear to act as hinders to engagement, psychologically and physically, in MBIs (Banerjee et al. 2017). A potential bidirectional relationship between mindfulness and aspects of TP (e.g., 
negative aspects of the past and future) could be evaluated using a cross-lagged panel design, involving repeated measurements of both sets of variables at multiple time points. Finally, the extent to which therapeutic techniques specifically aimed to reduce time perspective biases (Sword et al. 2014) also serve to increase mindfulness should be of interest in regard to the suggested influence from TP to mindfulness.

\section{Limitations and Future Research Directions}

Despite strengths of the present study, including the intervention-based approach and a comprehensive assessment of the future TP, limitations should be acknowledged. One concerns the lack of a control group in the first MBI. The effects of the second MBI were examined in an RCT study, which should control for a number of potential confounds. The wait-list control adopted in the present study might be considered less than ideal to isolate a mindfulness component, though, and future studies should consider active control groups aimed to pinpoint the effects unique to mindfulness training, preferably involving larger samples to permit evaluation of small effects. Moreover, in common with the bulk of prior mindfulness studies, female participants were overrepresented, which is of potential concern in regard to the generalizability of the findings. The results of a prior study indicating that women might be somewhat more responsive to this type of training, on average, than men (Rojiani et al. 2017). Nevertheless, we find no particular reason to suspect the association between mindfulness and time perspective, the topic in current focus, to be moderated by gender.

Future research should explore mechanism, whereby mindfulness is associated with particular aspects of time perspective, including links to persistent negative thought processes, such as rumination and worry. The relationship between mindfulness and a putative dynamic BTP component is another issue that awaits to be examined, something which would seem to require the use of some alternative method of measuring TP (Mello and Worrell 2015; Stolarski and Witowska 2017; Stolarski et al. 2015). Potential common associations of mindfulness and BTP with cognitive abilities are moreover of interest. Findings that better inhibition ability is related to lower DBTP (Zajenkowski et al. 2016) and is the executive component for which improvements following MBIs are most evident (Galliant 2016) are of interest at this point. Potential common neural underpinnings of the construct should be investigated further; preliminary evidence seem to suggest that both mindfulness (Tang et al. 2015) and BTP (Guo et al. 2017) involve increased connectivity of certain areas in the Default Mode Network. Finally, the suggestion that mindfulness training could be effective to reduce TP biases in clinical samples (Adshead 2013) needs to be evaluated in future studies.

Author Contributions MR designed the study, was responsible for the data analyses, and wrote a first draft of the paper. AK designed part of the study, collected parts of data, and collaborated in writing the final version of the manuscript. LG designed part of the study, collected parts of the data, and collaborated in the writing and editing of the final version of the manuscript. TÅ collaborated in the design of the study, the data analyses, and in writing and editing of the final version of the manuscript. MGC collaborated in the design of the study and in writing and editing the final version of the manuscript.

Funding The present research was supported by a grant to M. Rönnlund and M. G. Carelli from the Swedish Council for Research in the Humanities and Social Sciences (grant number 2015-02199).

Compliance with Ethical Standards The participants in this study provided informed consent prior to participation. The study protocols were approved by the regional ethics board in Umeå, Sweden.

Conflict of Interest The authors declare that they have no conflict of interest.

Informed Consent Informed consent was obtained from all participants included in the study prior to participation.

Open Access This article is distributed under the terms of the Creative Commons Attribution 4.0 International License (http:// creativecommons.org/licenses/by/4.0/), which permits unrestricted use, distribution, and reproduction in any medium, provided you give appropriate credit to the original author(s) and the source, provide a link to the Creative Commons license, and indicate if changes were made.

Publisher's Note Springer Nature remains neutral with regard to jurisdictional claims in published maps and institutional affiliations.

\section{References}

Adshead, G. (2013). The times of our lives: psychological disorders, time perception and the practice of mindfulness. European Journal of Psychotherapy and Counselling, 15(2), 139-150.

Aguado, J., Luciano, J. V., Cebolla, A., Serrano-Blanco, A., Soler, J., \& Garcia-Campayo, J. (2015). Bifactor analysis and construct validity of the five facet mindfulness questionnaire (FFMQ) in non-clinical Spanish samples. Frontiers in Psychology, 6, 404. https://doi.org/10. 3389/fpsyg.2015.00404.

Åström, E., Wiberg, B., Sircova, A., Wiberg, M., \& Carelli, M. G. (2014). Insights into features of anxiety through multiple aspects of psychological time. Integrative Psychology and Therapeutics, 2, 3. https:// doi.org/10.7243/2054-4723-2-3.

Baer, R. A., Hopkins, J., Krietemeyer, J., Smith, G. T., \& Toney, L. (2006). Using self-report assessment methods to explore facets of mindfulness. Assessment, 13(1), 27-45.

Baer, R. A., Smith, G. T., Lykins, E., Button, D., Krietemeyer, J., Sauer, S., \& Williams, J. M. (2008). Construct validity of the Five Facet Mindfulness Questionnaire in meditating and nonmeditating samples. Assessment, 15(3), 27-45. https://doi.org/10.1177/ 1073191107313003. 
Banerjee, M., Cavanagh, K., \& Strauss, C. (2017). Barriers to mindfulness: a path analytic model exploring the role of rumination and worry in predicting psychological and physical engagement in an online mindfulness-based intervention. Mindfulness. https://doi.org/ 10.1007/s12671-017-0837-4.

Bergomi, C., Tschacher, E., \& Kupper, Z. (2013). Measuring mindfulness: first steps towards the development of a comprehensive mindfulness scale. Mindfulness, 4(1), 18-32. https://doi.org/10.1007/ s12671-012-0102-9.

Bishop, S. R., Lau, M., Shapiro, S., Carlson, L., Anderson, N. D., et al. (2004). Mindfulness: a proposed operational definition. Clinical Psychology: Science and Practice, 11(3), 230-241.

Blomgren, A.-S., Svahn, K., Åström, E., \& Rönnlund, M. (2016). Coping strategies in late adolescence: relationships to parental attachment and time perspective. The Journal of Genetic Psychology, 177(3), 85-96. https://doi.org/10.1080/00221325.2016.1178101.

Boniwell, I., Osin, E., Lineley, A., \& Ivanchenko, G. V. (2010). A question of balance: time perspective and well-being in British and Russian samples. The Journal of Positive Psychology, 5(1), 24- 40.

Brown, K. W., \& Ryan, R. M. (2003). The benefits of being present: mindfulness and its role in psychological well-being. Journal of Personality and Social Psychology, 84(4), 822-848.

Carelli, M. G., Wiberg, B., \& Wiberg, M. (2011). Development and construct validation of the Swedish Zimbardo Time Perspective Inventory. European Journal of Psychological Assessment, 27(4), 220-227.

Coffey, K. A., Hartman, M., \& Fredrickson, B. L. (2010). Deconstructing mindfulness and constructing mental health: understanding mindfulness and its mechanisms of action. Mindfulness, 1, 235-253.

Cohen, J. (1988). Statistical power analysis for the behavioral sciences. New York (NY): Academy Press.

Cohen, S., Kamarck, T., \& Mermelstein, R. (1983). A global measure of perceived stress. Journal of Health and Social Behavior, 24, 385-396.

Desrosiers, A., Klemanski, D. H., \& Nolen-Hoeksema, S. (2013). Mapping mindfulness facets onto dimensions of anxiety and depression. Behavior Therapy, 44(3), 373-384. https://doi.org/10.1016/j. beth.2013.02.001.

Drake, L., Duncan, E., \& Sutherland, F. (2008). Time perspective and correlates of well-being. Time \& Society, 17(1), 95-107.

Droit-Volet, S., \& Heros, J. (2017). Time judgments as a function of mindfulness meditation, anxiety, and mindfulness awareness. Mindfulness, 8, 266-275.

Eskin, M., \& Parr, D. (1996). Introducing a Swedish version of an instrument measuring mental stress. Reports from the Department of Psychology, 813. Stockholm: Stockholm University, Department of Psychology.

Galliant, S. N. (2016). Mindfulness meditation practice and executive functioning: breaking down the benefit. Consciousness and Cognition, 40, 116-130. https://doi.org/10.1016/j.concog.2016.01.005.

Garland, E. L., Geschwind, N., Peteers, F., \& WIchers, M. (2015). Mindfulness training promotes upward spirals of positive affect and cognition: multilevel and autoregressive latent trajectory modeling analyses. Frontiers in Psychology, 6, 15. https://doi.org/10.3389/ fpsyg.2015.00015.

Guo, Y., Chen, Z., \& Feng, T. (2017). Neural substrates underlying balanced time perspective: a combined voxel-based morphometry and resting-state functional connectivity study. Behavioral Brain Research, 332, 237-242. https://doi.org/10.1016/j.bbr.2017.06.005.

Hansen, E., Lundh, L. G., Homman, A., \& Wångby-Lundh, M. (2009). Measuring mindfulness: pilot studies with the Swedish versions of the Mindful Attention Awareness Scale and the Kentucky Inventory of Mindfulness Skills. Cognitive Behavior Therapy, 38(1), 2-15. https://doi.org/10.1080/16506070802383230.

Janowski, K., \& Lucjan, P. (2012). Worry and mindfulness: the role in anxiety and depressive symptoms. European Psychiatry, 27(1), 133.
Kabat-Zinn, J. (1990). Full catastrophe living: using the wisdom of your body and mind to face stress, pain, and illness. New York, NY: Delacorte Press.

Keng, S.-L., Smoski, M. J., \& Robins, C. J. (2011). Effects of mindfulness on psychological health: a review of empirical studies. Clinical Psychology Review, 31(6), 1041-1056.

Khoury, B., Sharma, M., Rush, S. E., \& Fournier, C. (2015). Mindfulness-based stress reduction for healthy individuals: a metaanalysis. Journal of Psychosomatic Research, 78(6), 519-528. https://doi.org/10.1016/j.jpsychores.2015.03.009.

Lee, E. H. (2012). Review of the psychometric evidence of the Perceived Stress Scale. Asian Nursing Research, 6, 121-127. https://doi.org/ 10.1016/j.anr.2012.08.004.

Lilja, J. L., Frodi-Lundgren, A., Hanse, J. J., Josefsson, T., Lundh, L.-G., Broberg, S...., \& G, A. (2011). Five facets mindfulness questionnaire - reliability and factor structure: a Swedish version. Cognitive Behaviour Therapy, 40(4), 291-303. https://doi.org/10. 1080/16506073.2011.580367.

Mello, Z.-R., \& Worrell, F. C. (2015). The past, present, and the future: a conceptual model of time perspective in adolescence. In M. Stolarski, N. Fieualaine, \& W. van Beek (Eds.), Time perspective theory; review, research and application: essays in honor of Philip G. Zimbardo (pp. 115-129). New York: Springer.

Mooney, A., Earl, J. K., Mooney, C. H., \& Bateman, H. (2017). Using balanced time perspective to explain well-being and planning in retirement. Frontiers in Psychology, 8, 1781.

Morris, S. B. (2008). Estimating effect sizes from pre-posttest-control group designs. Organizational Research Methods, 11(2), 364-386. https://doi.org/10.1177/1094428106291059.

Muro, A., Felin-Soler, A., Castellà, J., Devi, J., \& Soler, J. (2017). Does time perspective predict life satisfaction? A study including mindfulness as a measure of time experience in a sample of Catalan students. Mindfulness, 8, 655-663. https://doi.org/10.1007/s12671-016-0644-3.

Papastamatelou, J., Unger, A., Giotakos, O., \& Athanasiadou, F. (2015). Is time perspective a predictor of anxiety and perceived stress? Some preliminary results from Greece. Psychological Studies, 60(4), 468477. https://doi.org/10.1007/s12646-015-0342-6.

Rojiani, R., Santoyo, J. F., Rahrig, H., Roth, H. D., \& Britton, W. B. (2017). Women benefit more than men in response to collegebased meditation training. Frontiers in Psychology, 8, 561.

Rönnlund, M., Åström, E., \& Carelli, M. G. (2017). Time perspective in late adulthood: age differences in past, present, and future dimensions, balance, and relations with subjective well-being. Timing and Time Perception, 5, 77-98. https://doi.org/10.1163/2213446800002081OAI.

Rönnlund, M., Åström, E., Adolfsson, R., \& Carelli, M. G. (2018). Perceived stress in adults aged 65 to 90 : relations to facets of time perspective and COMT Val158Met polymorphism. Frontiers in Psychology, 9, 378. https://doi.org/10.3389/fpsyg.2018.00378.

Schenström, O. (2017a). https://www.mindfulnesscenter.se/webshop/ekurser/haer-nu/

Schenström, O. (2017b). https://www.mindfulnesscenter.se/webshop/ekurser/mindfulness-och-medkaensla-med-sig-sjaelv-och-andra/

Scott, S. G., \& Bruce, R. A. (1995). Decision-making style: the development and assessment of a new measure. Educational and Psychological Measurement, 55(5), 818-831. https://doi.org/10. 1177/0013164495055005017.

Seema, R., \& Sircova, A. (2013). Mindfulness-a time perspective? Estonian study. Baltic Journal of Psychology, 14(1), 4-21.

Spijkerman, M. P. J., Pots, W. T. M., \& Bohlmeijer, E. T. (2016). Effectiveness of online mindfulness-based interventions in improving mental health: a review and meta-analysis of randomised controlled trials. Clinical Psychology Review, 45, 102-114. https://doi. org/10.1016/j.cpr.2016.03.009.

Stolarski, M., \& Cyniak-Cieciura, M. (2016). Balanced and less traumatized: balanced time perspective mediates the relationship between 
temperament and severity of PTSD syndrome in motor vehicle accident survivor sample. Personality and Individual Differences, 101, 456-461.

Stolarski, M., \& Matthews, G. (2016). Time perspectives predict mood states and satisfaction with life over and above personality. Current Psychology, 35(4), 516-526.

Stolarski, M., \& Witowska, J. (2017). Balancing one's own time perspective from aerial view: metacognitive processes in temporal framing. In A. Kostic \& D. Chadee (Eds.), Time perspective (pp. 117-141). London: Palgrave Macmillan.

Stolarski, M., Bitner, J., \& Zimbardo, P. G. (2011). Time perspective emotional intelligence and discounting of delayed awards. Time and Society, 20(3), 346-363. https://doi.org/10.1177/0961463X11414296.

Stolarski, M., Wiberg, B., \& Osin, E. (2015). Assessing temporal harmony: the issue of a balanced time perspective. In M. Stolarski, N. Fieualaine, \& W. van Beek (Eds.), Time perspective theory; review, research and application: essays in honor of Philip G. Zimbardo (pp. 57-71). New York: Springer.

Stolarski, M., Vowinckel, J., Jankowski, K. S., \& Zajenkowski, M. (2016). Mind the balance, be contended: balanced time perspective mediates the relationship between mindfulness and life satisfaction. Personality and Individual Differences, 93, 27-31.

Sword, R. M., Sword, R. K., Brunskill, S. R., \& Zimbardo, P. G. (2014). Time perspective therapy: a new time-based metaphor therapy for PTSD. Journal of Loss and Trauma, 19(3), 197-201.

Tang, Y. Y., Hözel, B. K., \& Posner, M. L. (2015). The neuroscience of mindfulness meditations. Nature Reviews Neuroscience, 16, 213 225. https://doi.org/10.1038/nrn3916.

Teasdale, J. D., \& Fogarty, S. J. (1979). Differential effects of induced mood on retrieval of pleasant and unpleasant events from episodic memory. Journal of Abnormal Psychology, 88, 248-257.

Vowinckel, J. C., Westerhof, G. J., Bohlmeijer, E. T., \& Webster, J. D. (2017). Flourishing in the now: initial validation of a present eudaimonic time perspective scale. Time \& Society, 26(2), 203226. https://doi.org/10.1177/0961463X15577277.

Walach, H., Buchheld, N., Buttensmüller, V., Kleinknecht, N., \& Schmidt, S. (2006). Measuring mindfulness-the Freiburg Mindfulness Inventory (FMI). Personality and Individual Differences, 40(6), 1543-1555. https://doi.org/10.1016/j.paid.2005.11.025.
Watkins, E., Moulds, M., \& Mackintosh, B. (2005). Comparisons between rumination and worry in a non-clinical population. Behavior Research and Therapy, 43, 1577-1585. https://doi.org/10.1016/j. brat.2004.11.008.

Webster, J. D., \& Ma, X. (2013). A balanced time perspective in adulthood: well-being and developmental effects. Canadian Journal on Aging, 32(4), 433-442. https://doi.org/10.1017/S0714980813000500.

Wittmann, M., Peter, J., Gutina, O., Otten, S., Kohls, N., \& Meissner, K. (2014). Individual differences in self-attributed mindfulness levels are related to the experience of time and cognitive self-control. Personality and Individual Differences, 64, 41-45.

Wittmann, M., Rudolph, T., Linares Gutierez, D., \& Winkler, I. (2015a). Time perspective and emotion regulation as predictors of age-related subjective passage of time. International Journal of Environmental Research and Public Health, 12, 16027-16042. https://doi.org/10. 3390/ijerph121215034.

Wittmann, M., Otten, S., Schötz, E., Sarikaya, A., Lehnen, H., Jo, H.-G., \& Meissner, K. (2015b). Subjective expansion of extended timespans in experienced meditators. Frontiers in Psychology, 5, 158. https://doi.org/10.3389/fpsyg.2014.01586.

Zajenkowski, M., Stolarski, M., Witowska, J., Marciantowicz, O., \& Lowicki, P. (2016). Fluid intelligence as a mediator of the relationship between executive control and balanced time perspective. Frontiers in Psychology, 7, 1844.

Zhang, J. W., Howell, R. T., \& Stolarski, M. (2013). Comparing three methods to measure a balanced time perspective: the relationship between a balanced time perspective and subjective well-being. Journal of Happiness Studies, 14(1), 1-16. https://doi.org/10.1007/ s10902-012-9322-x.

Zimbardo, P. G., \& Boyd, J. N. (1999). Putting time in perspective: a valid, reliable individual-differences metric. Journal of Personality and Social Psychology, 6, 1271-1288. https://doi.org/10.1037/0022-3514.77.6.1271.

Zimbardo, P. G., \& Boyd, J. N. (2008). The time paradox - the new psychology of time that will change your life. New York: Free Press.

Zimbardo, P. G., Sword, R., \& Sword, S. (2012). The time cure: overcoming PTSD with the new psychology of time perspective therapy. San Francisco: Jossey Bass.

Zimbardo, Z. M., McDermott, R., \& Zimbardo, P. G. (2017). A new measure of the expanded present time perspective. In A. Kostic \& D. Chadee (Eds.), Time perspective (pp. 41-62). London: Palgrave. 